\title{
HIV and aging: time to bridge the gap between clinical research and clinical care
}

\author{
Fátima Brañas ${ }^{1} \cdot$ Giovanni Guaraldi ${ }^{2} \cdot$ Matilde Sánchez-Conde $^{3}$
}

Received: 12 December 2018 / Accepted: 9 January 2019 / Published online: 17 January 2019

(c) European Geriatric Medicine Society 2019

Considered separately, aging and HIV are unquestionably hot topics. The amount of scientific evidence focused on each one is huge, growing and unmanageable, because new fields to investigate are suggested daily and the knowledge we possess is probably just the tip of the iceberg. The more we know, the more still remains for us to learn. The study of HIV can help to better understand the process of aging, and the study of aging sheds light on the way HIV impacts the organism. However, putting them together (HIV and aging), interest has been triggered and a new horizon has been opened in terms of research and clinical care.

Some special issues have been developed in recent years focused on different aspects of "HIV and aging," all of them published in HIV-specialized journals. However, as far as we know, this is the first one in a geriatric medicine journal, which makes it unique. In this issue, European Geriatric Medicine invited experts on HIV, experts on aging and experts on HIV and aging to build this project specifically aimed at geriatricians. Through 12 special articles, the authors address how the HIV scenery has dramatically changed in developed countries, from survival to quality of life, from predominantly younger people to the predominantly older population, to focus on the complexity of older adults living with HIV. Comorbidity, polypharmacy and geriatric syndromes are significant new problems to take into account in the clinical management of these patients. Cognitive impairment and stigma were present from the beginning of the pandemic, so they are old problems that require a new approach in the era of the aging HIV population. One

Fátima Brañas

fatima.branas@salud.madrid.org; fbranas@gmail.com

1 Geriatrics Department, Infanta Leonor University Hospital, Avda. Gran Vía del Este 80, 28031 Madrid, Spain

2 Modena HIV Metabolic Clinic, University of Modena and Reggio Emilia, Modena, Italy

3 Infectious Diseases and HIV Department, Ramón y Cajal University Hospital, Madrid, Spain article reviews four European cohorts of older HIV adults and another the biomarkers of aging in HIV. However, two articles were written specifically to convince geriatricians to get involved in the care of older adults with HIV: "Why are people with HIV considered 'older adults' in their fifties?" is the first one [1]. The authors focus on the core concept of biological age instead of chronological age to consider someone as older or not. This is a very geriatric idea in theory. All geriatricians understand that there should not be an age cutoff point to make decisions in terms of health and that function and quality of life should be the key. However, they always think about the upper cutoff point-the oldest of older individuals - while sometimes being very restricted in terms of the lower cutoff point, and if the patient is not 75 years old or older, he or she is "too young for geriatricians" independently of his/her physical function, frailty status or health-adverse event risk-ultimately, independently of his/her biological age, centered on his/her chronological age.

Two clinical cases should be useful to illustrate this issue (F.B. unpublished data 2017). A woman with breast cancer was referred for comprehensive geriatric assessment (CGA) to the geriatrician before surgery. She suffered from hypertension, chronic venous disease and pathology of the left rotator cuff tendon. She was under first-step analgesics and no more pills. In the CGA, she walked independently without any help and was able to perform basic, instrumental and advanced activities of daily living. Her walk speed was $1.29 \mathrm{~m} / \mathrm{s}$, her short physical performance battery (SPPB) score was 12 and her hand grip strength was $21.2 \mathrm{~kg}$ (BMI 25). From a cognitive point of view, her MOCA test score was 27, and her score on the Geriatric Depression Scale Short Form (GDS-SF) was 3. Her Mini-Nutritional Assessment Short Form (MNA-SF) score was 13. She lived alone. She was in touch with her children and took care of her grandchildren every day. The second clinical case is a man who was referred to a geriatrician for a global evaluation. He had been living with HIV for 18 years. He also suffered 
from $\mathrm{HCV}$, which was treated and cured. He had a history of migraines, anxiety, insomnia, dorsal herpes zoster and condylomas. He was on-demand painkillers, three benzodiazepines, amitriptyline and antiretroviral treatment. In the evaluation, he walked independently without any help and was able to perform basic activities of daily living. He performed some instrumental activities of daily living very limited by pain. His walk speed was $0.58 \mathrm{~m} / \mathrm{s}$, his SPPB score was 12 and his hand grip strength was $24.8 \mathrm{~kg}$ (BMI 25). From a cognitive point of view, his MOCA test score was 25 and his score on the GDS-SF was 12. His MNA-SF score was 10 . He lived alone without any social contacts. The lady was 82 years old. The man was 61 years old. The lady was fit and robust and did not need any specific intervention from the geriatrician. The man was frail, in pain and suffered from some geriatric syndromes such as polypharmacy, depression, malnutrition risk and social fragility, which require specific and global intervention from a geriatrician. These are just two cases, two real-life cases, but what we want to highlight is that chronological age is not a good marker of biological aging taking into account the lower cutoff point as well. HIV infection has been postulated as a model of accelerated aging [2-4], due to the persistent and chronic activation of the immune system that leads to immune exhaustion and accelerated immunosenescence, even when on optimal immuno-virological control treatment. The clinical expression is an increased prevalence of aging-related non-HIVassociated comorbidities $[5,6]$ and a rising prevalence of frailty occurring earlier than in the general population [7-9]. Thus, people living with HIV (PLWH) seem to be biologically older than their chronological age.

The second article is titled "HIV-Geriatric Medicine: the geriatrician's role" [10]. In this one, the authors underline the core that constitutes the geriatrician's specific expertise and suggest putting it toward the service of complex older adults with HIV to bring value with the development of CGA integrated in a multidisciplinary team led by HIV specialists. As they point out, most older adults with HIV nowadays are robust and do not need a geriatrician's intervention, but one in four suffer from geriatric syndromes and, specifically, functional impairment [7,11-13], and this $25 \%$ will be surely benefit from a geriatrician's evaluation through the CGA.

No evidence exists of how the care of older adults with HIV should specifically be provided, but something has to be done to bridge the gap between clinical research and clinical care. Many researchers are publishing about frailty, physical function and/or geriatric syndromes in PLWH, which is crucial to provide expansive and solid scientific evidence, but very few are implementing the findings in clinical care, in real life [14].

In our opinion, working together of medical specialties, such as in orthogeriatrics, cardiogeriatrics or oncogeriatrics, is crucial to establish geriatric HIV medicine. This makes sense because of the complexity of aging PLWH. New patients with new needs require new care models. Wedoctors and health systems - have to adapt ourselves and our work dynamics to the patients, and not the other way around. Our work makes sense if it provides answers to the problems real patients face. Thirty-seven years ago, a new unknown devastating disease appeared in our world, bringing death, stigma and suffering. In the scientific community, some people viewed it as a problem they did not want to be involved in, but others, a small group, were able to see it as a challenge and worked hard to bring the answers those patients needed. Today, thanks to them, we are talking about the way PLWH are aging. It is important that HIV specialists and geriatricians are able to face the challenge presented by the current reality; we cannot turn a blind eye. The majority of PLWH are older adults, and it is mandatory to focus on their function to help them achieve healthy aging and better quality of life.

\section{Compliance with ethical standards}

Conflict of interest FB has received honoraria for the following: MISP grants from MSD; speaking at symposia organized on behalf of MSD and ViiV Healthcare; developing educational materials for MSD; and board membership from ViiV Healthcare. GG received research grant from Gilead sciences, ViiV, MERCK and Janssen. From the same companies he accepted travel sponsorship and speaker honorarium. He attended advisory boards of Gilead sciences, ViiV and MERCK. MSC has received honoraria for the following: speaking at symposia organized on behalf of MSD, ViiV Healthcare and Gilead.

Ethical approval This article does not contain any studies with human participants or animals performed by any of the authors.

Informed consent For this type of study formal consent is not required.

\section{References}

1. Sánchez-Conde M, Díaz-Álvarez J, Dronda F, Brañas F (2018) Why are people with HIV considered "older adults" in their fifties? Eur Geriatric Med. https://doi.org/10.1007/s4199 9-018-0148-x

2. Pathai S, Bajillan H, Landay AL, High KP (2014) Is HIV a model of accelerated or accentuated aging? J Gerontol Ser A Biol Sci Med Sci 69(7):833-842

3. Jimenez Z, Sanchez-Conde M, Branas F (2018) HIV infection as a cause of accelerated aging and frailty. Revista Espanola de Geriatria y Gerontol 53(2):105-110

4. Cohen J, Torres C (2017) HIV-associated cellular senescence: a contributor to accelerated aging. Ageing Res Rev 36:117-124

5. Guaraldi G, Orlando G, Zona S, Menozzi M, Carli F, Garlassi E et al (2011) Premature age-related comorbidities among HIVinfected persons compared with the general population. Clin Infect Dis 53(11):1120-1126 
6. Smit M, Brinkman K, Geerlings S, Smit C, Thyagarajan K, Sighem A et al (2015) Future challenges for clinical care of an ageing population infected with HIV: a modelling study. Lancet Infect Dis 15(7):810-818

7. Brañas F, Jiménez Z, Sánchez-Conde M, Dronda F, López-Bernaldo De Quirós JC, Pérez-Elías MJ et al (2017) Frailty and physical function in older HIV-infected adults. Age Ageing 14:1-5

8. Guaraldi G, Brothers TD, Zona S, Stentarelli C, Carli F, Malagoli A et al (2015) A frailty index predicts survival and incident multimorbidity independent of markers of HIV disease severity. AIDS 29(13):1633-1641

9. Greene M, Covinsky KE, Valcour V, Miao Y, Madamba J, Lampiris $\mathrm{H}$ et al (2015) Geriatric syndromes in older HIV-infected adults. J Acquir Immune Defic Syndr 69(2):161-167

10. Brañas F, Ryan P, Troya J, Sánchez-Conde M (2018) GeriatricHIV medicine: the geriatrician's role. Eur Geriatric Med. https:// doi.org/10.1007/s41999-018-0144-1

11. Erlandson KM, Schrack JA, Jankowski CM, Brown TT, Campbell TB (2014) Functional impairment, disability, and frailty in adults aging with HIV-infection. Curr HIV/AIDS Rep 11(3):279-290
12. Erlandson KM, Allshouse AA, Rapaport E, Palmer BE, Wilson CC, Weinberg A et al (2015) Physical function impairment of older, HIV-infected adults is associated with cytomegalovirus immunoglobulin response. AIDS Res Hum Retrovir 31(9):905-912

13. Greene M, Covinsky K, Astemborski J, Piggott DA, Brown T, Leng $S$ et al (2014) The relationship of physical performance with HIV disease and mortality. AIDS 28(18):2711-2719

14. Siegler EL, Burchett CO, Glesby MJ (2018) Older people with HIV are an essential part of the continuum of HIV care. J Int AIDS Soc 21(10):e25188

Publisher's Note Springer Nature remains neutral with regard to jurisdictional claims in published maps and institutional affiliations. 